Cette rubrique est dirigée par

L. LANIER

Ingenieur en chef du G.R.E.F.

Professeur à l'École nationale

du Gènie rural, des Eaux et des Forèts

14, rue Girardet

54042 NANCY CEDEX

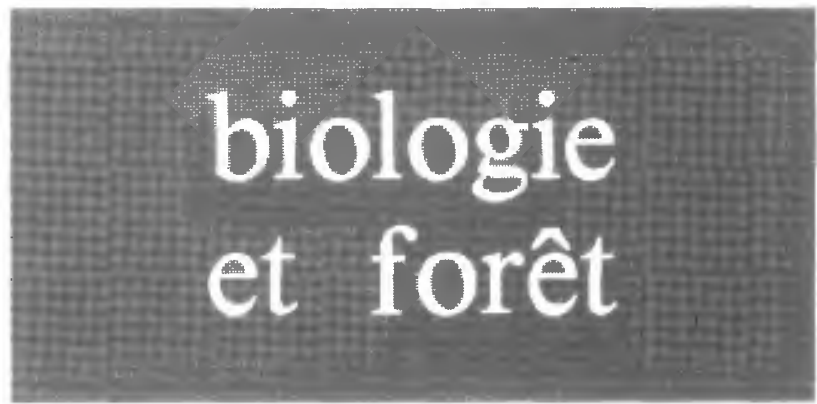

\title{
LA SÉCHERESSE DE 1976 : \\ INFLUENCE DES DÉFICITS HYDRIQUES SUR LA CROISSANCE DES ARBRES FORESTIERS
}

G. AUSSENAC

Il faut qu'il se produise un phénomène de l'ampleur de la sécheresse de 1976 pour que l'on réalise pleinement l'importance du facteur eau pour les arbres forestiers. II s'agit en effet d'un facteur que l'on a tendance à oublier dans les climats humides de la France, en dehors de la région méditerranéenne.

En réalité, l'eau représente partout un facteur primordial de la production forestière (Pardé 1966, Aussenac 1973, Aussenac-Ducrey 1974, Aussenac 1975).

L'année 1976, par les extrêmes de sécheresse qu'elle a atteint, a été dans bien des cas catastrophique; il est cependant possible de profiter de ces circonstances exceptionnelles pour essayer d'améliorer notre connaissance de l'écologie des essences et des peuplements forestiers.

\section{LA SÉCHERESSE DE 1976}

Sans citer de longues séries de chiffres, il nous semble quand même utile de rappeler les caractères généraux de cette sécheresse. Ce phénomène a concerné l'ensemble du pays, à l'exception du Sud-Est ; il a été particulièrement grave au nord d'une ligne Bordeaux-Belfort.

Le déficit pluviométrique s'est installé dès le mois de décembre 1975 et a duré jusqu'à la fin du mois d'août 1976. La figure $n^{\circ} 1$ donne les écarts de précipitations par rapport à la normale (1931-1960) pour les périodes du $1^{\text {er }}$ décembre 1975 au 31 août 1976 et du $1^{\text {er }}$ décembre 1975 au 30 septembre 1976.

On peut remarquer les grands écarts atteints jusqu'en tin août dans le Bassin Parisien, la Bretagne et une partie de la Normandie $(-60 \%)$. En septembre, les écarts ont diminué d'une façon générale. 


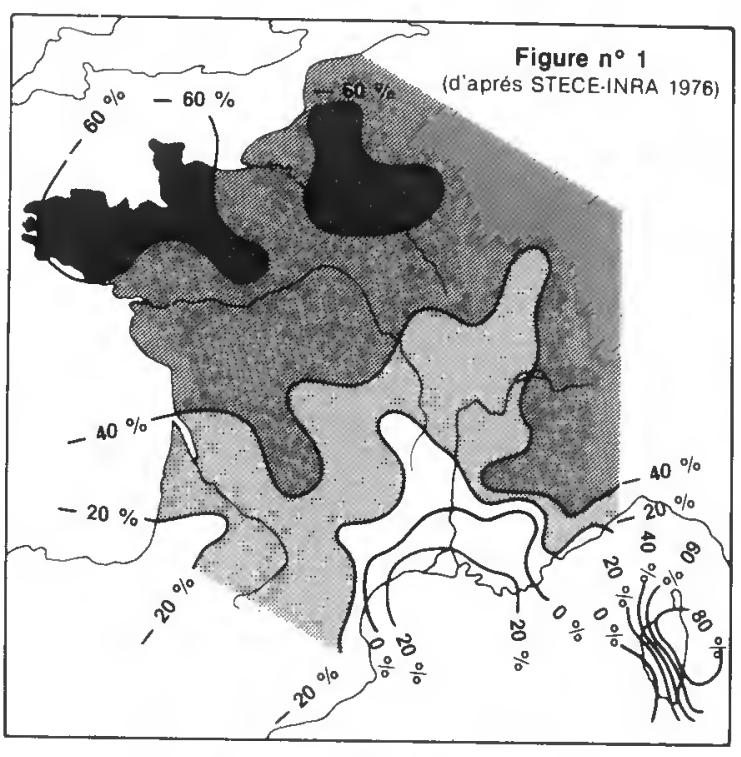

Période du 01.12.75 au 31.08.76

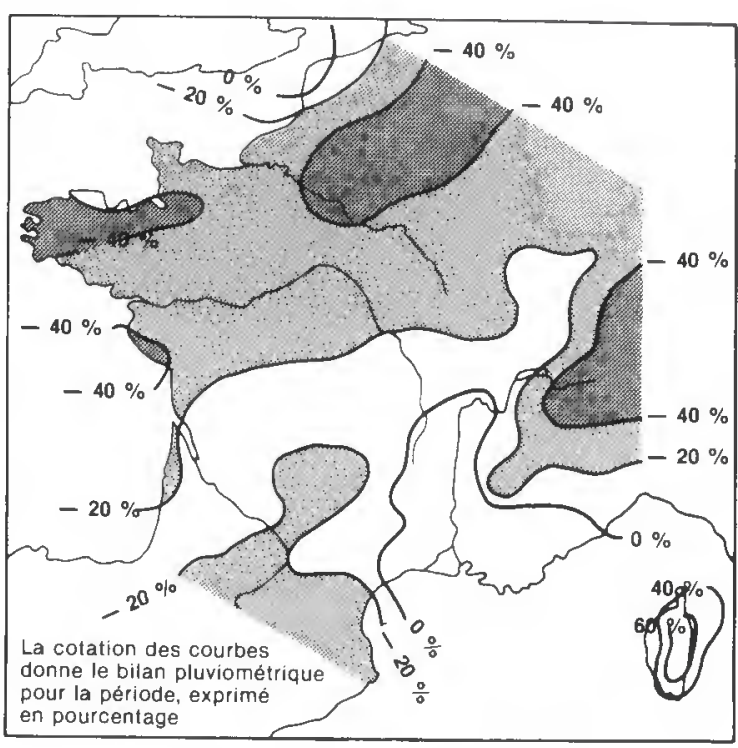

Période du 01-12-75 au 30-09-76

Quels ont été les effets de ce déficit hydrique exceptionnel? Pour essayer de répondre, nous avons effectué une enquête auprès de l'Office national des forêts et du Service des forêts ( ${ }^{1}$ ). Nous disposons aussi des observations effectuées dans les dispositifs expérimentaux du département "Forêts" de l'Institut national agronomique (I.N.R.A.). Une enquête de ce type est toujours difficile et ne peut prétendre à être exhaustive. II faut donc interpréter les informations recueillies avec beaucoup de précautions. On se réfèrera à ce sujet aux bonnes études de Monnier (1976) et de Marion (1976). Nous ferons simplement apparaître les grandes tendances. Les zones les plus touchées ont été l'Ouest de la France, le Bassin Parisien, la Lorraine, la Franche-Comté et le Centre. Ce sont effectivement les régions qui ont été les plus affectées par les déficits pluviométriques.

Au cours de l'été 1976, les plantations de l'automne 1975 et du printemps 1976 ont énormément souffert. Les dégâts ont été considérables (mort d'une partie ou de la totalité de l'arbre) et généralisés chez toutes les essences (feuillus ou résineux). La mortalité a été importante et a atteint fréquemment $100 \%$. II apparaît clairement qu'elle a été spécialement forte sur les sols superficiels.

Dans les peuplements plus anciens (5 à 10 ans), les dégâts ont été moins sérieux. Ils sont en corrélation étroite avec les conditions de station: type de sol, importance du couvert.

Dans certains cas, les arbres en situation de coupe d'abri ou d'abri latéral (bandes, trouées) se sont bien comportés et ont bien résisté à la sécheresse. 
Dans les peuplements âgés (50 ans et plus), les dommages ont été dans l'ensemble plus ponctuels : mort d'arbres isolés en lisière ou à l'intérieur des peuplements. II semble que la plupart des essences ait été concernée, à des degrés divers, par ces phénomènes. Pour certairês espèces (bouleaux, charmes), on a noté une chute importante de feuilles en juillet et en août, sans pour autant qu'il y ait eu mort de l'arbre. Des dessechements de la cime se sont produits chez le hêtre.

Les plantations de l'année ou celles âgées de deux à trois ans mises à part, les essences de reboisement les plus touchées par la sécheresse sont les suivantes: les mélèzes (Larix decidua, Larix leptolepis), l'épicéa commun (Picea abies), l'épicéa de Sitka (Picea sitchensis), le douglas vert (Pseudotsuga menziesii), cette dernière particulièrement en Sologne.

Des dégâts importants ont aussi été signalés chez les pins : Pinus sylvestris et Pinus nigra dans des stations défavorables à sols très superficiels. Notons aussi parmi les espèces particulièrement atteintes par la sécheresse, bien qu'elles soient peu utilisées en reboisement: Chamaecyparis lawsoniana, Thuja plicata, Tsuga heterophylla. En 1977, un certain nombre d'arbres ont péri de façon isolée dans les peuplements.

En résumé, l'ensemble des données recueillies permet de faire un certain nombre de remarques :

- les dégâts de la sécheresse ont été plus importants dans les jeunes plantations que dans les peuplements déjà constitués ou même dans les vieux peuplements ;

- les dégâts ont été graves sur les sols superficiels, caillouteux ou sableux;

- enfin, il apparaît clairement que les espèces exotiques ont été beaucoup plus affectées que les essences indigènes.

En dehors de ces phénomènes visibles, il y a eu, en 1976, une perte d'accroissement qu'il est cependant difficile de chiffrer.

\section{APERÇU SUR LE FONCTIONNEMENT DU COMPLEXE SOL-PLANTE-ATMOSPHĖRE}

La sécheresse de 1976 par sa gravité exceptionnelle a révélé de façon claire l'ensemble des composantes à prendre en considération pour interpréter les différents phénomènes observés : la composante climatique (pluviométrie et évapotranspiration), la composante sol (réserve hydrique) et enfin la composante biologique (nature et âge de l'arbre, structure du peuplement). En fait, c'est l'ensemble sol-arbre-atmosphère qui doit être pris en compte si l'on veut analyser le comportement de l'arbre face aux différentes conditions hydriques qui se présentent. Sans aborder des aspects trop théoriques, il est intéressant d'étudier ces différentes composantes et le fonctionnement du système sol-plante-atmosphère.

\section{Le climat}

Le climat intervient dans le cycle de l'eau par les précipitations qui constituent les apports et par l'évapotranspiration, phénomène qui est lui-même sous la dépendance d'autres facteurs cli. matiques (énergie radiative, température et humidité de l'air, vent).

L'évapotranspiration climatique est appelée évapotranspiration potentielle (E.T.P.). On l'a définie comme l'évapotranspiration d'un couvert végétal fermé et parfaitement alimenté en eau. L'évapotranspiration réelle (E.T.R.) du couvert est inférieure ou égale à l'évapotranspiration poten- 


\section{G. AUSSENAC}

tielle. On peut la définir à partir du bilan d'énergie ${ }^{\left({ }^{2}\right.}$ du couvert dont un des termes correspond précisément à l'énergie nécessaire à la vaporisation de l'eau. L'évapotranspiration réelle dépend de facteurs climatiques tels que le rayonnement net, le déficit hygrométrique de l'air, le vent, des caractéristiques propres au couvert végétal et de la disponibilité en eau du sol.

Au niveau local, certaines structures de peuplement permettent de modifier les paramètres cli-

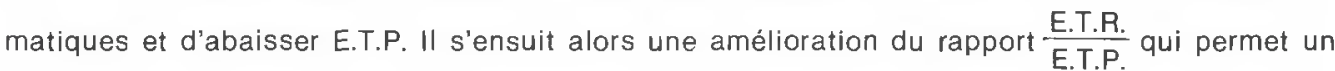
état hydrique très favorable à la croissance par diminution des fréquences de stress hydrique. Ces effets interviennent lorsque pour les jeunes peuplements (régénération naturelle ou artificielle) on utilise des structures plus ou moins fermées : coupe d'abri, clairières ou bandes forestières. Les expériences menées à Nancy de 1967 à 1973 (Aussenac 1975) montrent clairement l'influence bénéfique de certaines structures de peuplements pour la croissance des arbres dans leur jeune âge. Les observations effectuées en 1976, dans plusieurs régions de France, montrent d'ailleurs que les arbres ont mieux résisté dans ces types de plantations.

\section{Le sol}

Le sol constitue le réservoir qui stocke l'eau utilisable par les plantes. On admet généralement que l'eau disponible pour les végétaux correspond à la quantité d'eau comprise entre deux niveaux d'humidité correspondants à deux niveaux d'énergie de liaison de l'eau (potentiel de l'eau $\psi$ ) : la capacité au champ $\left(0,63\right.$ atmosphère, soit un $\left.\mathrm{pF}\left({ }^{3}\right)=2,8\right)$ et le point de flétrissement permanent (16 atmosphères, $\mathrm{pF}=4,2$ ) qui correspond au niveau de sécheresse au-delà duquel les plantes ne peuvent plus extraire l'eau du sol et meurent. On sait cependant maintenant que les arbres peuvent subsister au-delà du $\mathrm{pF}=4,2$.

La quantité d'eau disponible dépend du sol et de l'enracinement. L'importance de ce dernier est conditionnée par les caractéristiques propres à l'arbre (espèce, âge), mais aussi par les caractéristiques du sol (texture, structure, profondeur). La quantité d'eau disponible pour les végétaux est appelée : réserve utile (R.U.).

La figure $n^{\circ} 2$ donne, en exemple pour Nancy, l'évolution théorique de la réserve en eau du sol en 1976 pour trois valeurs de réserve utile : $150 \mathrm{~mm}, 100 \mathrm{~mm}$ et $50 \mathrm{~mm}$. On voit que la date d'apparition de la sécheresse se situe à la mi-mai pour une réserve de $50 \mathrm{~mm}$, à la mi-juin pour une réserve de $100 \mathrm{~mm}$ et à la fin juin pour une réserve de $150 \mathrm{~mm}$. En réalité, l'évolution de la réserve en eau du sol évolue assez différemment, car seule une fraction (on l'estime aux 2/3) de l'eau utile est facilement accessible pour les végétaux, c'est la réserve facilement utilisable. Au-delà, l'eau devient de plus en plus difficile à extraire: c'est ce qui explique que l'évolution réelle de la réserve en eau du sol est différente du calcul théorique.

La réserve utile peut être très variable selon les cas. Elle peut représenter (en pourcentage du poids de terre sèche) par exemple : pour un sol sableux : $5 \%$; pour un sol limoneux : 10 à $15 \%$; pour un sol argilo-limoneux : 12 à $20 \%$.

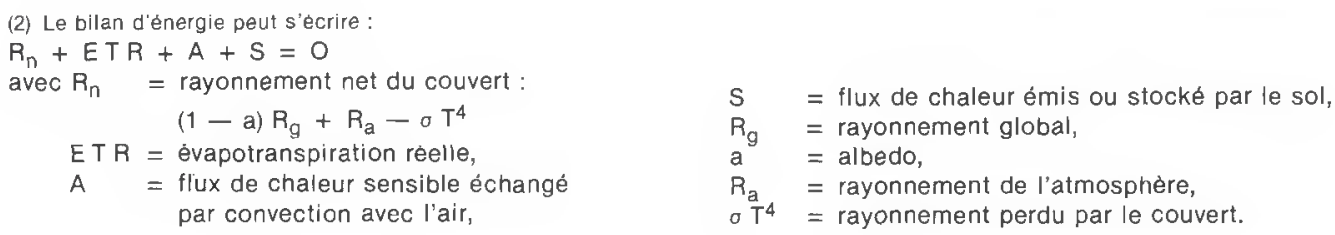

(3) Le $p F=\log _{10} w$. La valeur du $p F$ a la capacite au champ varie avec la nature et la structure du sol : 1,3 pour des sols très sableux, 3,4 pour des sols très argileux. 


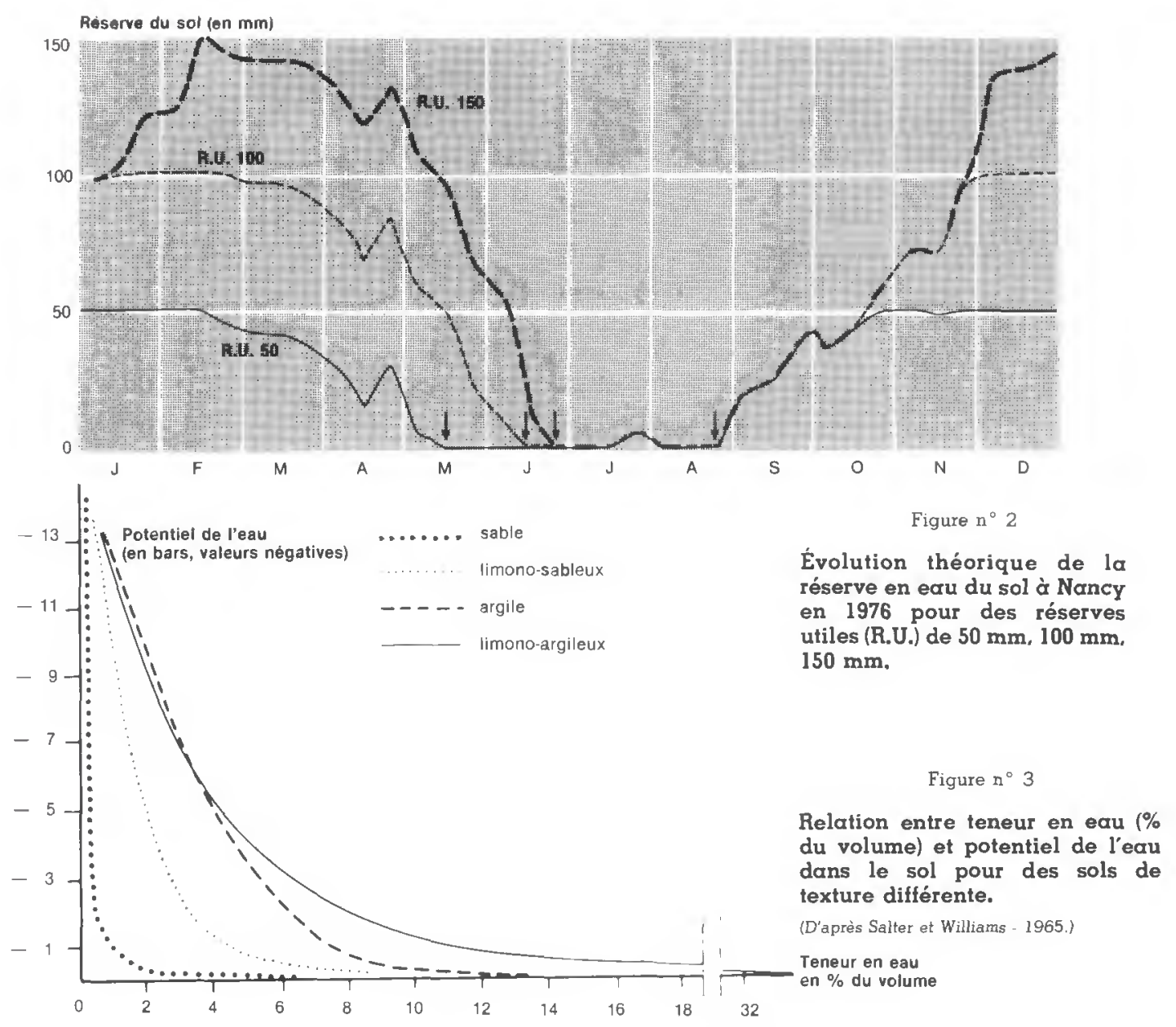

La détermination des réserves en eau utile peut être faite $\left({ }^{4}\right)$ tranche par tranche de sol (par exemple $10 \mathrm{~cm}$ ) et l'on peut la calculer pour l'ensemble du profil. Ainsi, pour un sol de $100 \mathrm{~cm}$ d'épaisseur, la réserve utile d'un sol sableux sera égale à $60 \mathrm{~mm}$, alors que pour un sol argilolimoneux, elle atteindra $240 \mathrm{~mm}$.

De grosses différences existent donc quant à la hauteur d'eau disponible selon les types de sol, d'autres différences existent si l'on se place dans une perspective dynamique, c'est-à-dire la perspective du transfert de l'eau du sol dans l'arbre.

Lorsque la teneur en eau du sol commence à être réduite dans un sol argileux, la disponibilité réelle en eau est moins bonne que dans un sol sableux. II en résulte un frein à l'extraction de l'eau par les végétaux dans les sols argileux, ce qui a pour conséquence de prolonger la durée du stock d'eau. Dans un sol sableux, le débit d'eau est plus grand, mais il sera finalement plus court. La figure $n^{\circ} 3$ montre l'évolution des potentiels de l'eau dans le sol en fonction des teneurs en eau (en pourcentage du volume du sol). On voit que dans un sol limono-argileux, il

(4) Pour calcuier la reserve, on emploie la formule

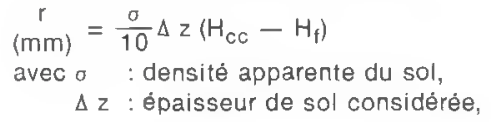

$H_{\mathrm{CC}}$ : humidité du sol à la capacité au champ,

$H_{f}$ : humidité au point de flétrissement permanent. 


\section{G. AUSSENAC}

y a une diminution très progressive des potentiels (donc une augmentation lente de la sécheresse); par contre, dans un sol sableux, les potentiels de l'eau sont relativement stables lorsque la teneur en eau diminue à partir de la capacité au champ, puis brusquement une faible variation de teneur en eau suffit à faire baisser très fortement le potentiel; en d'autres termes, il y a alors soudainement une forte augmentation de la sécheresse.

Le réservoir sol apparaît donc ici jouer son rôle non seulement par "ses dimensions ", mais aussi par la dynamique de ses disponibilités en eau. Toutes ces caractéristiques sont à prendre en compte dans les problèmes de créations de peuplements forestiers au moment du choix des essences et des types de traitements (coupe rase, coupe par bande, etc.).

\section{L'arbre}

Le végétal est pour nous, plus précisément, l'arbre qui constitue l'interface entre le sol qui, on l'a vu, peut assurer une plus ou moins bonne alimentation en eau, et l'atmosphère qui exerce une demande plus ou moins grande.

Dans le transport de l'eau vers l'atmosphère, l'arbre ne se comporte pas comme une mèche inerte. Des mécanismes de régulation du flux d'eau existent et réduisent plus ou moins la transpiration. Cette régulation se fait essentiellement grâce aux stomates qui peuvent être plus ou moins ouverts ${ }^{5}$ ). Lorsque les stomates sont complètement fermés, il subsiste une transpiration faible, mais encore notable qui s'exerce au travers de la cuticule des feuilles. Cette transpiration cuticulaire est fortement réduite chez les arbres des régions méditerranéennes. La chute des feuilles constitue aussi un phénomène permettant de réduire les pertes en eau.

La circulation de l'eau dans le système sol-plante-atmosphère a été modélisée en s'appuyant sur des analogies électriques ( $\left.{ }^{6}\right)$.

Selon les cas, le potentiel de l'eau peut varier : dans le sol de 0 à -16 bars el même à - 30 bars; dans l'arbre de -2 à -40 bars et dans l'atmosphère de 0 à -28 bars (à une température de $25{ }^{\circ} \mathrm{C}$ pour une humidité relative de $98 \%$ ) ou même à -1700 bars (pour une température de $25{ }^{\circ} \mathrm{C}$ et pour une humidité relative de $30 \%$ ).

Les arbres présentent des différences notables de comportement dans leur mobilisation de l'eau du sol. Les Pins se distinguent assez nettement des autres résineux. La figure $n^{\circ} 4$ relative à des semis (Lopushinsky 1969) est à cet égard intéressante. Elle montre, pour Pinus ponderosa et Abies grandis, l'évolution de la transpiration dans le temps lorsqu'il y a accroissement de la sécheresse du sol. On remarque que Pinus ponderosa a une transpiration relativement stable pendant plusieurs jours, qui brusquement décroît et se stabilise pour des valeurs faibles. A ce moment-là, la valeur du potentiel de base $(7)$ atteint - 17,6 bars. Chez Abies grandis (Lopushinsky indique qu'il en est de même chez Pseudotsuga menziesii) par contre, la transpiration baisse progressivement pour finalement se stabiliser à des valeurs très faibles, le potentiel de base étant alors de $-25,8$ bars.

Sur des arbres plus âgés en peuplements, Aussenac et Granier (1978) (figure $n^{\circ}$ 5) observent aussi des différences de comportement entre les pins (Pinus sy/vestris, Pinus nigra) et Pseudotsuga menziesii.

(5) La variatıon du degré d'ouverture des stomates est sous la dépendance de mécanismes physiques et chimiques.

(6) On peut écrire $T=\frac{\Delta \psi}{\mathrm{R}}=\frac{\psi \text { sol }-\psi \text { feuille }}{\mathrm{R} \text { sol }+\mathrm{A} \text { arbre }}$

$\mathrm{T}$
$\psi$ sol $\quad$ : potentiel de l'eau dans le sol,

w feuille : potentiel de l'eau dans la feuille,

$R$ sol : résistance au transiert de l'eau dans le sol,

$R$ arbre : résistance au transfert de l'eau dans l'arbre

Ce type d'analogie n'est valable que dans l'hypothése d'un flux conservatif, c'est-à-dire quand la transpiration est égale à l'absorption.

(7) Potentiel de base : potentiel de l'eau dans l'arbre mesuré en fin de nuit avant le lever du solell. 

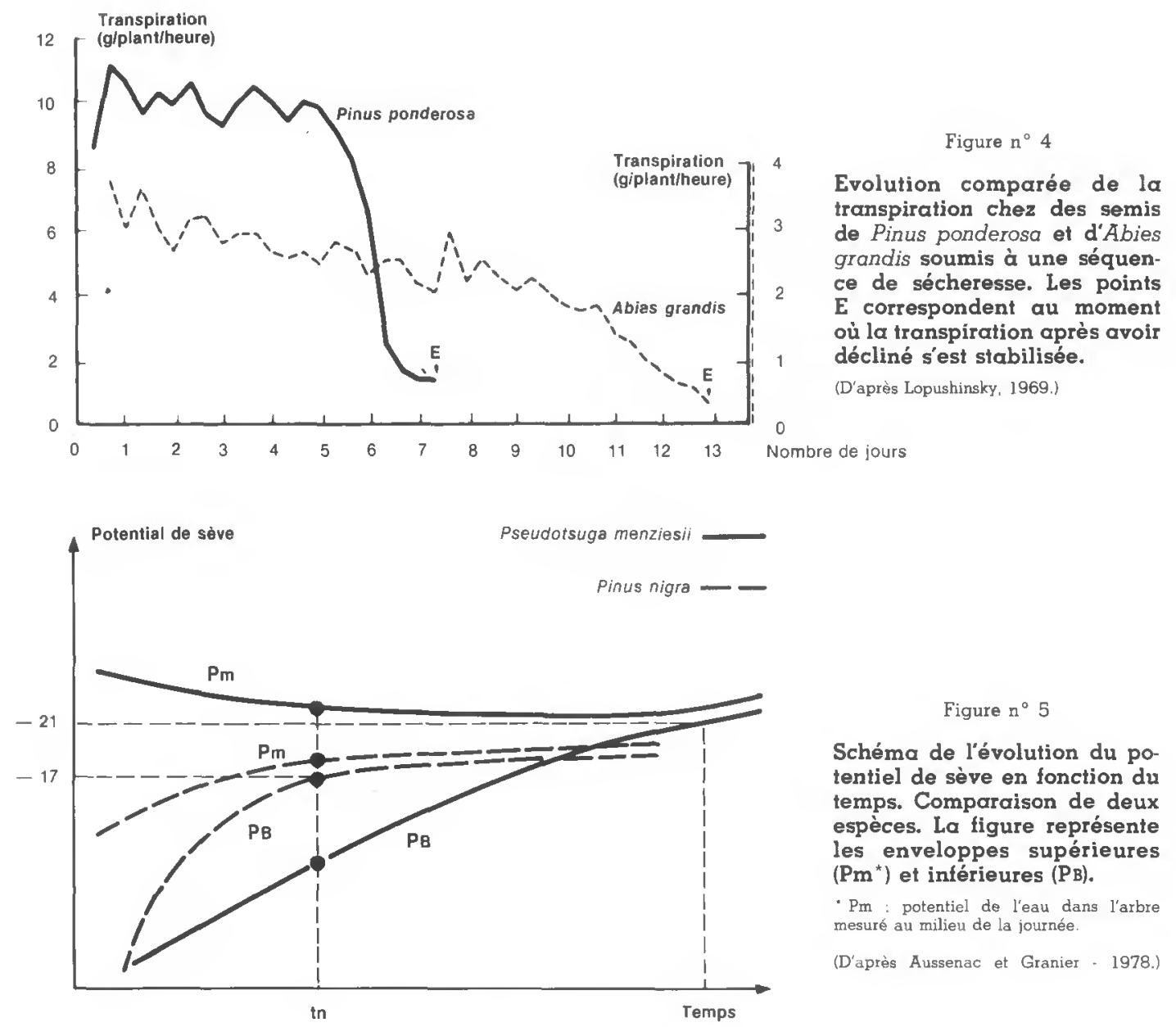

Figure $n^{\circ} 5$

Schéma de l'évolution du potentiel de sève en fonction du temps. Comparaison de deux espèces. La figure représente les enveloppes supérieures $\left(\mathrm{Pm}^{*}\right)$ et inférieures $(\mathrm{PB})$.

- Pm : potentiel de l'eau dans l'arbre mesuré au milieu de la journée.

(D'après Aussenac et Granier - 1978.)

L'interprétation de ces phènomènes (Aussenac-Granier, 1977) est la suivante :

Chez les pins, la transpiration et la photosynthèse sont élevées quand l'alimentation en eau est correcte. Lorsque la sécheresse s'installe, il y a diminution rapide de la transpiration et de la photosynthèse avec un blocage de ces activités pour des potentiels de base relativement forts : - 16,0 à - 17,0 bars. A partir de ce moment, la transpiration n'est plus que cuticulaire et les déperditions d'eau sont très faibles, l'arbre résiste bien à la sécheresse. Au contraire, le Douglas continue à transpirer et donc à photosynthétiser jusqu'à des potentiels hydriques bas : - 21 bars. Le blocage de son activité physiologique n'intervient donc qu'à des potentiels beaucoup plus faibles. Ces caractéristiques font du Douglas un arbre qui a de grandes facultés d'assimilation phytosynthétique, mais elles peuvent aussi le mettre en difficulté lorsque la dis. ponibilité en eau devient trop faible. Or, nous avons déjà dit que, dans les sols sableux, la disponibilité en eau diminuait brusquement : on comprend alors que, dans ce type de sol, les essences comme le Douglas ou le Sapin de Vancouver puissent se trouver dans une situation hydrique très défavorable, ce qui peut avoir pour conséquence des dessèchements brutaux et parfois mortels. Les pins par contre vont avoir un meilleur comportement, car ils ont freiné beaucoup plus tôt leur consommation en eau. La conséquence sylvicole évidente de cette ana- 

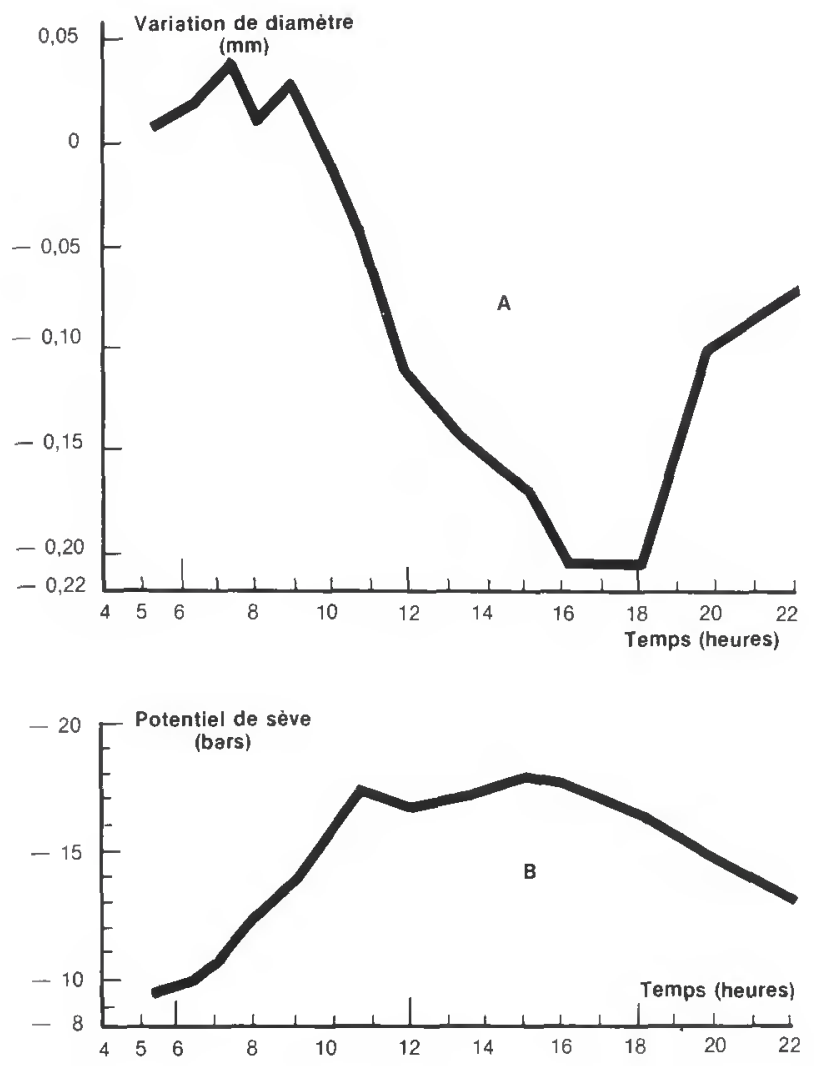

Figure $n^{\circ} 6$

Variation du diamètre diun rameau de Douglas (Pseudotsuga menziesii) (A) et variation de la pression de sève du même arbre (B) au cours d'une journée.

(D'après Zaerr - 1971, in Granier - 1977.)

lyse écophysiologique est la suivante : sur les sols sableux, il faut éviter des essences qui ont le comportement du Douglas et du sapin de Vancouver.

En fait, en période de sécheresse, chez le Douglas et le sapin de Vancouver, la baisse de la transpiration est associée à une fermeture stomatique faible si on la compare à celle qui intervient chez les pins. On peut interpréter ce phénomène comme la résultante d'une régulation stomatique peu sensible aux conditions de sécheresse et d'une bonne régulation de la transpiration à l'intérieur de la feuille.

Chez les arbres adultes, les recherches ont montré qu'une quantité non négligeable d'eau pouvait être stockée dans le xylème des racines, du tronc et des branches, permettant à l'arbre de tamponner les demandes en eau. Ce phénomène est à mettre en relation avec le rétrécissement du tronc que l'on peut observer dans le courant de la journée (tigure $n^{\circ} 6$ ). Running et al (1975) estiment que dans un peuplement mûr de Douglas, l'eau ainsi mise à disposition représente $60 \mathrm{~mm}$ qui viennent s'ajouter à la réserve utile du sol. Au niveau d'un arbre individuel dans une placette située en Orégon, dans la chaîne des Cascades, ils estiment le stockage égal à 4300 litres pour un arbre de $80 \mathrm{~m}$ de haut et 3,2 litres pour un arbre d'une hauteur de $2 \mathrm{~m}$. Selon Running et al, ces stockages très différents auraient pour conséquence des périodes de stress différentes, par exemple pour une même année en une même station, 17 jours pour l'arbre le plus grand et 54 jours pour l'arbre le plus petit.

\section{INFLUENCES DES DÉFICITS HYDRIQUES SUR LA CROISSANCE}

L'effet des déficits hydriques sur la croissance est difficile à analyser dans la nature, car en même temps que le manque d'eau, interviennent aussi d'autres iacteurs climatiques : rayonnement global, température, hygrométrie relative, autant de facteurs qui peuvent influencer plus ou moins directement les différentes activités biologiques de l'arbre (transpiration, photosynthèse, croissance, mise à fleurs, etc.), elles-mêmes s'influençant mutuellement. Le modèle de Fritts (1966) (figure $n^{\circ} 7$ ) montre comment une réduction des précipitations aboutit à une réduction de l'épaisseur d'un cerne; il n'est cependant pas complet, car il ne prend pas en compte la totalité des mécanismes physiologiques. 


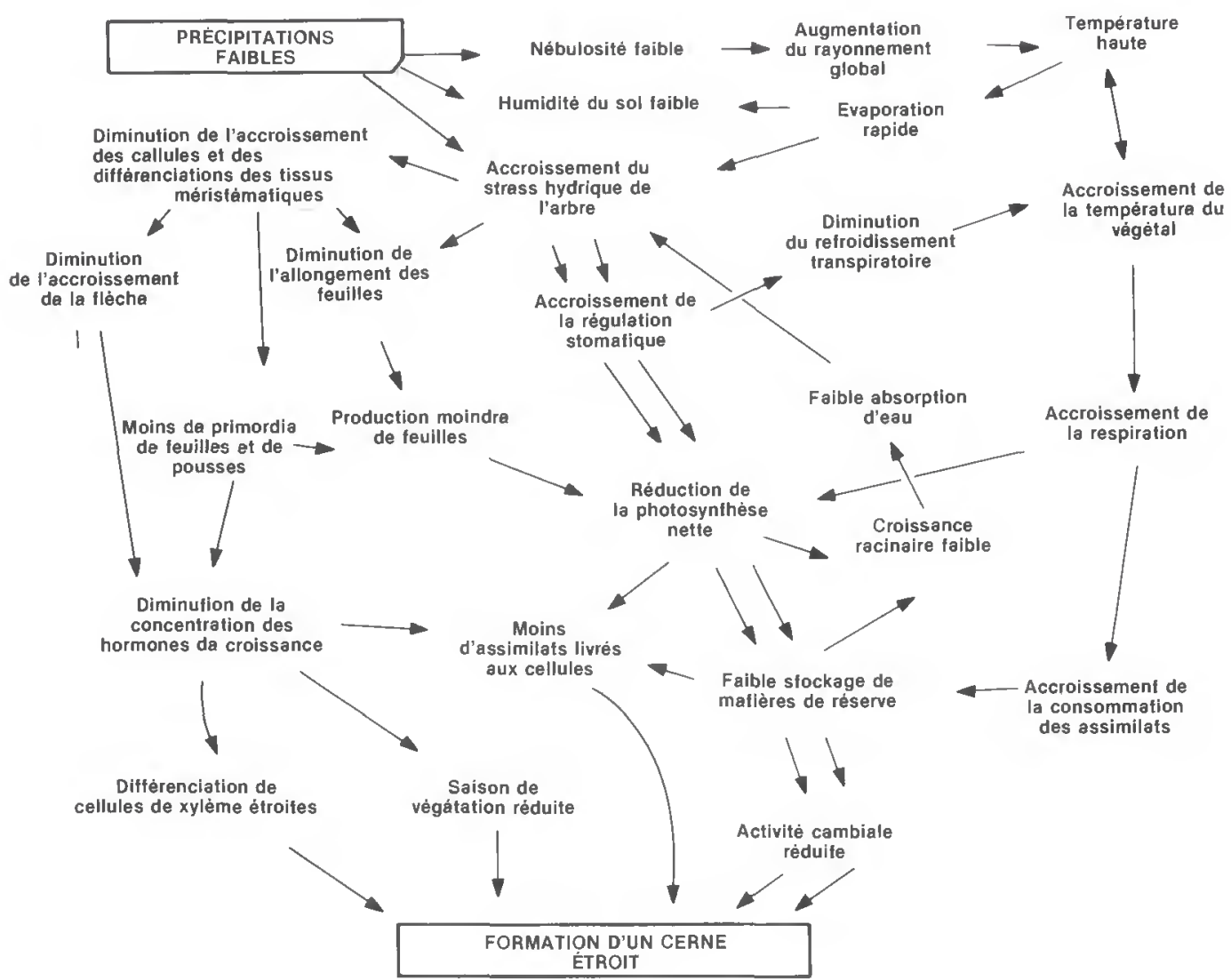

Figure $n^{\circ} 7$

Influence de la réduction des précipitations sur l'épaisseur du cerne (d'après Fritt's 1966)

La photosynthèse nette est attectee plus ou moins fortement par la sécheresse qui s'accompagne d'une fermeture des stomates et d'une perte d'activité des chloroplastes. On ne connaît pas la part respective de ces deux phénomènes dans la diminution de l'assimilation photosynthétique.

La régulation stomatique (et donc une moindre disponibilité en eau) paraît être responsable de la restriction de la photosynthèse dans le cas de sécheresse se produisant avec de très forts éclairements; dans les autres cas, il semble que cela soit la décroissance de l'activité chloroplastique qui intervienne.

La baisse de photosynthèse résulte aussi dans certains cas de la limitation de l'accroissement de la surface foliaire et de l'accélération de la sénescence des feuilles.

On écrit souvent que la croissance en hauteur d'une année déterminée est affectée uniquement par les conditions hydriques de l'année précédente, alors que la croissance en diamètre serait influencée par les conditions hydriques de l'année considérée. En réalité, les phénomènes sont 
plus compliqués que cela (Aussenac, 1973). L'importance et les modalités d'installation de la sécheresse interviennent en interaction avec le comportement écophysiologique propre de l'espèce.

Au laboratoire, il est possible de séparer l'action des différents facteurs sur la croissance. On a ainsi pu montrer par exemple que la sécheresse pouvait influencer le nombre et la taille des aiguilles. On a aussi montré que, chez les semis, la croissance en hauteur était affectée par des sécheresses même légères. Ainsi, chez Pinus taeda et Pinus echinata, Stransky et Wilson (1964), ont montré que la croissance en hauteur était affectée par des potentiels d'eau dans le sol de l'ordre de -2 à -3 bars $(\mathrm{pF}=3,3 \cdot 3,4)$. II semble d'ailleurs que ce niveau de $\mathrm{pF}$ où se manifestent des réductions de croissance soit assez général chez les résineux. Selon les espèces et selon les modalités (prècoce ou tardive), la sécheresse peut affecter la croissance racinaire, ce qui finalement se répercute sur la croissance de la partie aérienne l'année suivante. Ces phénomènes ont été observés par Becker (1977) chez Abies alba, Abies nordmanniana et Alba numidica.

Dans les peuplements, l'étude de l'influence des déficits hydriques sur la croissance est difficile et la plupart du temps, on procède par recherche de corrélation. Les travaux de Zahner et Donnelly (1967) sont à cet égard intéressants (figure $n^{\circ} 8$ ). II montre comment l'action combinée des conditions hydriques de l'année en cours et celles de l'année précédente sont fortement

Figure $n^{\circ} 8$

Relations entre la largeur du cerne annuel chez deux plantations de Pinus resinosa. La pluviométrie est égale à la somme de la pluviométrie du 16 mai au 15 septembre d'une année. plus la pluviométrie du lor juillet au 31 août de l'année précédente. Le déficit hydrique est égal à la somme du déficit du $l^{\text {er }}$ juin au 30 septembre d'une année. plus le déficit du 16 août au 30 septembre de l'année précédente.

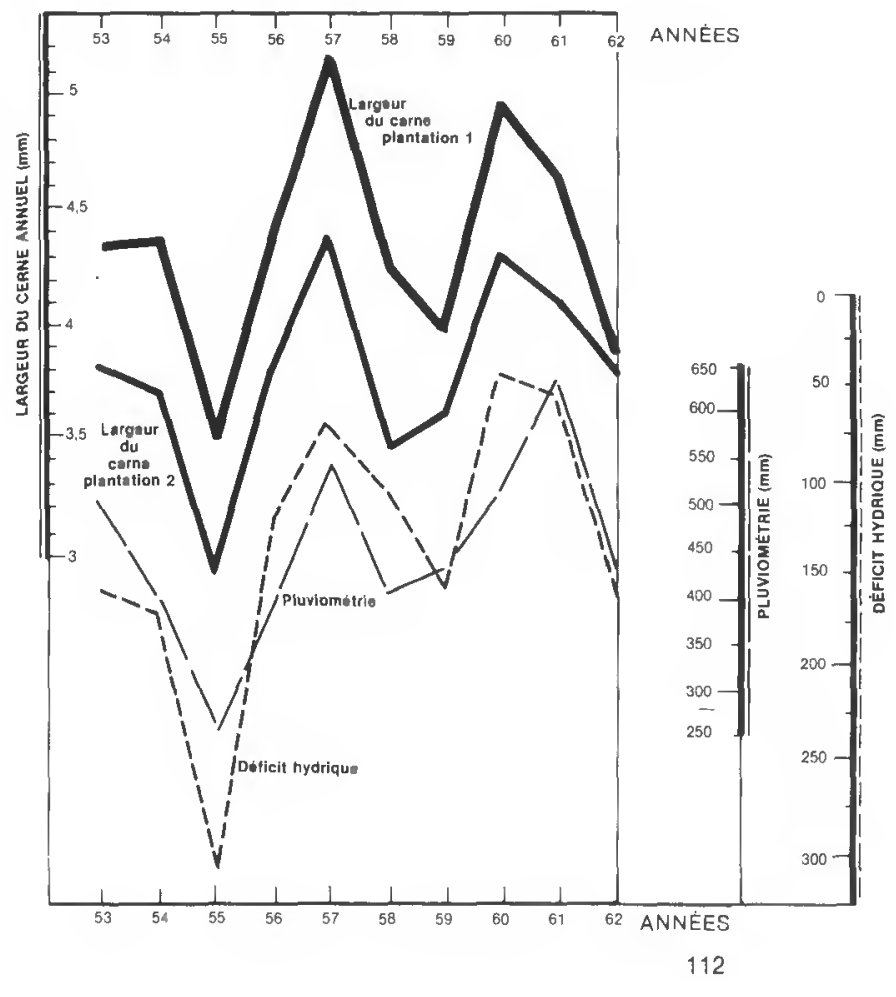

(D'après Zahner et Donnelly - 1967, in Zahner - 1968.) 
corrélées avec l'accroissement du cerne. En conditions expérimentales chez Pinus sylvestris, Polge et Keller (1968) trouvent un effet positif de l'irrigation sur la largeur du cerne. Dans ce domaine, les recherches sont ouvertes, car les influences peuvent varier avec l'espèce, les conditions de station et le moment de la saison de végétation où se situe la sécheresse.

\section{CONCLUSION}

La sécheresse de 1976 par sa gravité a placé au premier plan le rôle essentiel de l'alimentation hydrique pour le bon fonctionnement de l'arbre.

L'influence des conditions d'alimentation en eau sur la biologie des arbres est encore relativement méconnue. Dans ce domaine, des progrès considérables sont encore à faire par la recherche pour améliorer nos connaissances sur le fonctionnement hydrique des essences et proposer une bonne adaptation des espèces ou provenances introduites aux conditions locales de stations.

Au niveau de la pratique forestière, la prise en compte d'un certain nombre de résultats scientifiques peut d'ores et déjà, dans quelques cas, permettre de mieux adapter essences et conditions de stations.

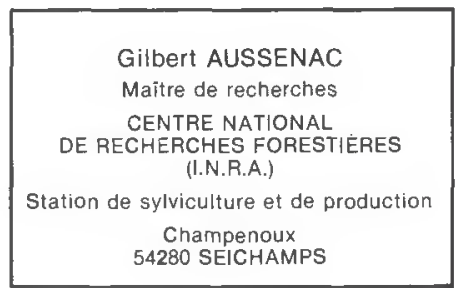

\section{BIBLIOGRAPHIE}

AUSSENAC (G.). - Climat, microclimat et production ligneuse. Annales des sciences forestieres, vol. 29 , $n^{\circ} 3,1973$, pp. 239-258.

AUSSENAC (G.), DUCREY (M.). - A propos de l'arboretum de Royat. Climatologie et potentialités de production forestière. Revue forestière française, $\mathrm{n}^{\circ} 6,1974$, pp. 447-458.

AUSSENAC (G.). - Couverts forestiers et facteurs du climat : leurs Interactions, conséquences écophysiologiques chez quelques résineux. - Thèse Docteur ès-sciences. Nancy, 1975. - $234 \mathrm{p}$.

AUSSENAC (G.), GRANIER (A.). - Quelques résultats de cinétique journalière du potentiel de séve chez les arbres forestiers. Annales des sciences forestieres, vol. 35, $n^{\circ} 1,1978, p p .19-32$.

BECKER (M.). - Contribution à l'étude de la transpiration et de l'adaptation à la sécheresse des jeunes plants résineux. Exemple de trois sapins du pourtour méditerranéen: Abies alba, Abies nordmanniana, Abies numidica. Annales des sciences forestières, vol. 34, n², 1977, pp. 137-158.

FRITTS (H.C.). - Growth rings of trees : their correlation with climate. Sciences, $n^{\circ} 154,1966$, p. 973.

GRANIER (A.). - Transtert de sève brute dans les troncs des arbres. Aspects méthodologique et physiologique. Annales des sciences forestières, vol. 34, n 1, 1977, pp. 17-45.

LOPUSHINSKY (W.). - Stomatal closure in conifer seedlings in response to leaf moisture stress. Botanical gazette, vol. 130, n4, 1969, pp. 258-263.

MARION (J.E.). - Quelques conséquences de la sécheresse pour les sylviculteurs. Bullefin de vulgarisation forestière, $n^{\circ} 76 / 9,1976$.

MONNIER (Y.). - Les conséquences de la sécheresse. Bulletin d'information de l'Office national des forêts, $n^{\circ} 40,1976$, pp. $10-16$. 


\section{G. AUSSENAC}

PARDÉ (J.). - Forêt et reboisement à haute productivité en France. Revue forestière française, $n^{\circ} 11,1966$, pp. 718-724.

POLGE (H.), KELLER (R.). - Influence de l'approvisionnement en eau sur la structure interne des accroissements annuels. Annales des sciences forestieres, vol. 25, $n^{\circ} 3,1968$, pp. 125-133.

RUNNING (S.W.), WARING (R.H.), RYDELL (R.A.). - Physiological control of water flux in Conifers. A computer simulation model. Oecologia, vol. 18, 1975, pp. 1-16.

STRANSKY (J.L.), WILSON (D.R.). - Terminal elongation of loblolly and short leaf pine seedlings under soil moisture stress. Proceedings, Soil science society of America, vol. 28, $n^{\circ} 3,1964$, pp. 439-440.

ZAHNER (R.), DONNELLY (J.R.). - Refining correlations of rainfall and radial growth in young red pine. Ecology, vol. 48, 1967 , p. 525 .

ZAHNER (R.). - Water deficits and growth of trees. In : Kozlowski (T.T.) Ed. - Water deficits and plant growth (vol. Il). - Academic Press, New York and London, 1968, pp. 135-185.

\section{ERRATUM}

Dans l'article: "Quelques données statistiques sur les premières gelées d'automne en France ", de G. AUSSENAC, (Revue forestière française, $n^{\circ} 6$, 1977) une erreur s'est glissée dans le tableau $n^{\circ}$ 2, $1^{\text {re }}$ colonne : il faut supprimer "Mont-de-Marsan" à la date du 12 octobre et rajouter " 2 octobre: Mont-de-Marsan".

\section{$\begin{array}{lllllllllllllllll}\text { I } & N & F & O & R & M & A & T & \text { I } & O & N & S\end{array}$}

La Revue forestière française a été heureuse d'apprendre l'attribution à M. Jean POURTET, ingé: nieur général du G.R.E.F. (e.r.) du "Grand Prix d'Agriculture de la ville de Paris ".

Cette brillante distinction est parfaitement méritée par la haute compétence acquise par M. POURTET en matière de dendrologie, et spécialement de populiculture, et par le souci, qui a toujours été le sien, de mettre ses connaissances au service de l'enseignement, de la recherche et de l'administration. La réputation dont jouissent ses travaux à l'étranger fait honneur à la science forestière française.

Ce prix lui a été remis, le 14 février dernier, par le représentant de $M$. le Maire de Paris, à l'occasion d'une réunion à l'Hôtel de Ville.

Nous avons le plaisir de lui adresser nos bien vives félicitations. 